\title{
Projective Lag Synchronization of Delayed Neural Networks Using Intermittent Linear State Feedback
}

\author{
Junjian Huang, ${ }^{1,2}$ Chuandong Li, ${ }^{1,3}$ Tingwen Huang, ${ }^{4}$ Huaqing Li, ${ }^{1}$ and Mei Peng ${ }^{5}$ \\ ${ }^{1}$ College of Computer Science, Chongqing University, Chongqing 400030, China \\ ${ }^{2}$ Department of Computer Science, Chongqing University of Education, Chongqing 400067, China \\ ${ }^{3}$ School of Electronics and Information Engineering, Southwest University, Chongqing 400715, China \\ ${ }^{4}$ Texas A\&M University at Qatar, P.O. Box 23874, Doha, Qatar \\ ${ }^{5}$ College of Mathematical and Computer Science, Yangtze Normal University, Chongqing 400084, China
}

Correspondence should be addressed to Chuandong Li; licd@cqu.edu.cn

Received 12 August 2013; Accepted 10 September 2013

Academic Editor: Jinde Cao

Copyright (C) 2013 Junjian Huang et al. This is an open access article distributed under the Creative Commons Attribution License, which permits unrestricted use, distribution, and reproduction in any medium, provided the original work is properly cited.

The problem of projective lag synchronization of coupled neural networks with time delay is investigated. By means of the Lyapunov stability theory, an intermittent controller is designed for achieving projective lag synchronization between two delayed neural networks systems. Numerical simulations on coupled Lu neural systems illustrate the effectiveness of the results.

\section{Introduction}

In the past few years, synchronization of neural networks has been extensively investigated due to their successful application in many areas, such as communication, modeling brain activity, signal processing, and combinatorial optimization. There are several different synchronization schemes including complete, lag, projective, generalized, phase, and anticipated synchronization [1-9]. In projective synchronization, the master-slave systems can be synchronized up to a scaling factor. Due to the potential applications in secure communication, projective synchronization has attracted increasing attention [10-13]. In [10], the authors study the projective synchronization for different chaotic delayed neural networks via sliding mode control approach. Function projective synchronization of two-cell QuantumCNN chaotic oscillators using adaptive method is investigated in [11]. It is worth noting that the propagation delay may exist in remote communication systems. However, to the best of the authors' knowledge, few results (if any) for the projective lag synchronization of neural networks with time delay have been reported in the literature.

In this paper, we will deal with the analysis issue for projective lag synchronization of neural networks with time delay by intermittent control approach. Recently, we have employed this method to stabilize and synchronize chaotic systems [14-16]. In this paper, by using Lyapunov stability theory and intermittent control technique, the intermittent controllers and corresponding parameter update rules are designed to obtain projective lag synchronization of neural networks. The rest of the paper is organized as follows. In Section 2, we formulate the problem of projective lag synchronization of coupled neural networks. In Section 3, a general scheme for the projective lag synchronization is presented. Numerical simulations are given in Section 4. Finally, conclusions are given in Section 5.

\section{Problem Formulation and Preliminaries}

In this paper, we consider the chaotic cellular neural networks described by

$$
\begin{gathered}
\dot{x}_{i}(t)=-c_{i} x(t)+\sum_{j=1}^{n} a_{i j} f_{i}(x(t))+\sum_{j=1}^{n} b_{i j} g_{i}(x(t-\tau)), \\
i=1,2,3, \ldots, n ; t>0, \\
x(t)=\varphi(t), \quad-\tau \leq t \leq 0,
\end{gathered}
$$


or, in a compact form,

$$
\begin{gathered}
\dot{x}(t)=C x(t)+A f(x(t))+B g(x(t-\tau)), \quad t>0, \\
x(t)=\varphi(t), \quad-\tau \leq t \leq 0,
\end{gathered}
$$

where $x(t)=\left[x_{1}(t), x_{2}(t), \ldots, x_{n}(t)\right] \in R^{n}$ denotes the state vector, $C, A$, and $B \in R^{m \times m}$ are constant matrices, $\tau$ is the time delay, and $f, g: R^{m} \rightarrow R^{m}$ are nonlinear functions satisfying the Lipschitz condition, namely; there exist positive constants $L_{f}, L_{g}$ such that, for all $x, y \in R^{n}$,

$$
\begin{aligned}
& |f(x)-f(y)| \leq L_{f}|x-y| \\
& |g(\alpha)-g(\beta)| \leq L_{g}|\alpha-\beta| .
\end{aligned}
$$

Consider the corresponding slave system given in the following form:

$$
\begin{gathered}
\dot{y}(t)=C y(t)+A f(y(t))+B g(y(t-\tau))+u(t), \\
t>0, \\
y(t)=\psi(t), \quad-\tau \leq t \leq 0,
\end{gathered}
$$

where $y(t) \in R^{n}$ denotes the state vector, $C, A$, and $B \in$ $R^{n \times n}$ are constant matrices, and $u(t)$ denotes the intermittent feedback control defined as follows:

$$
u(t)= \begin{cases}H(t)+K(t), & n T \leq t<n T+\sigma T, \\ H(t), & n T+\sigma T \leq t<(n+1) T\end{cases}
$$

where $k$ denotes the control strength, $0<\sigma<1$ denotes the switching rate, $T$ denotes the control period, and $H(t)$ and $K(t)$ are the active control functions.

Let $\theta$ be the transmittal delay. Defining the projective lag synchronization error between systems (2) and (4) as $e(t)=$ $y(t)-\alpha x(t-\theta)$, where $\alpha$ denotes projective scaling factor, we have the following error dynamical system:

$$
\begin{aligned}
\dot{e}(t)= & \dot{y}(t)-\alpha \dot{x}(t-\theta) \\
= & C y(t)+A f(y(t))+B g(y(t-\tau))+u(t) \\
-\alpha(C x(t-\theta)+A f(x(t-\theta)) & \\
& +B g(x(t-\tau-\theta))) .
\end{aligned}
$$

Under the control of the form (5), the system (6) can be rewritten as

$$
\begin{array}{r}
\dot{e}(t)=C y(t)+A f(y(t))+B g(y(t-\tau))+H(t)+K(t) \\
-\alpha(C x(t-\theta)+A f(x(t-\theta))+B g(x(t-\tau-\theta))), \\
n T \leq t<n T+\sigma T, \\
\dot{e}(t)=C y(t)+A f(y(t))+B g(y(t-\tau))+H(t) \\
-\alpha(C x(t-\theta)+A f(x(t-\theta))+B g(x(t-\tau-\theta))), \\
n T+\sigma T \leq t<(n+1) T .
\end{array}
$$

Definition 1. The master system (2) and the slave system (4) are said to be projective lag synchronization if there exist a compact set $\alpha, \sigma$ and delay time $\theta$ such that, for any initial values $\varphi\left(t_{0}\right), \psi\left(t_{0}\right) \in \Omega, t_{0} \in[-\tau, 0]$, the error system is exponentially stable; that is,

$$
\begin{array}{r}
\lim _{t \rightarrow \infty}\|e(t)\|=\lim _{t \rightarrow \infty}\|y(t)-\alpha x(t-\theta)\| \leq\|e(0)\| e^{-\sigma t}, \\
\forall t \geq 0 .
\end{array}
$$

\section{Main Results}

This section addresses the projective lag synchronization problem of coupled neural networks.

Theorem 2. Suppose that there exist constants $\alpha$, the coupling strength $k$, time delay $\theta$, and the functions $H(t), K(t)$ such that

(i) $C+C^{T}-2 k I+g_{1} I \leq 0$;

(ii) $C+C^{T}-g_{2} I \leq 0$;

(iii) $H(t)=-A f(y(t))-B g(y(t-\tau))+\alpha A f(x(t-\theta))+$ $\alpha B g(x(t-\tau-\theta))$;

(iv) $K(t)=-k(y(t)-\alpha x(t-\theta))$;

(v) $g_{1} \sigma-(1-\sigma) g_{2}>0$.

Then, the projective lag synchronization error system (7) is globally exponentially stable, that is; the projective lag synchronization between the master system (2) and the slave system (4) under intermittent control (5) is achieved.

Proof. Consider the following Lyapunov function:

$$
V(t)=e(t)^{T} e(t) .
$$

When $n T \leq t<n T+\sigma T$, the derivative of (9) with respect to time $t$ along the trajectories of the first subsystem of the system (7) is calculated and estimated as follows:

$$
\begin{aligned}
& \dot{V}(t)=2 e(t)^{T} \dot{e}(t) \\
&=2 e(t)^{T}[ C y(t)+A f(y(t))+B g(y(t-\tau)) \\
&+H(t)+K(t) \\
&-\alpha(C x(t-\theta)+A f(x(t-\theta)) \\
&+B g(x(t-\tau-\theta)))] \\
&=e(t)^{T}\left[C+C^{T}-2 k I+g_{1} I\right] e(t)-g_{1} e(t)^{T} e(t) \\
& \leq-g_{1} e(t)^{T} e(t) .
\end{aligned}
$$

Similarly, when $n T+\sigma T \leq t<(n+1) T$, one obtains

$$
\begin{aligned}
\dot{V}(t)= & 2 e(t)^{T} \dot{e}(t)=2 e(t)^{T} \\
\times & {[C y(t)+A f(y(t))+B g(y(t-\tau))+H(t)} \\
& \quad-\alpha(C x(t-\theta)+A f(x(t-\theta))+B g(x(t-\tau-\theta)))] \\
= & e(t)^{T}\left[C+C^{T}-g_{2} I\right] e(t)+g_{2} e(t)^{T} e(t) \\
\leq & g_{2} e(t)^{T} e(t) .
\end{aligned}
$$


Therefore,

$$
\begin{gathered}
\dot{V}(t) \leq-g_{1} V(t), \quad n T \leq t<n T+\sigma T, \\
\dot{V}(t) \leq g_{2} V(t), \quad n T+\sigma T \leq t<(n+1) T .
\end{gathered}
$$

Then, one observes that

$$
\begin{array}{r}
V(t) \leq\|V(n T)\| \exp \left(-g_{1}(t-n T)\right), \\
n T \leq t<n T+\sigma T, \\
V(t) \leq\|V(n T+\sigma T)\| \exp \left(g_{2}(t-n T-\sigma T)\right), \\
n T+\sigma T \leq t<(n+1) T .
\end{array}
$$

By (12) and (13), we can obtain the following.

(1) For $0 \leq t<\sigma T$,

$$
\begin{gathered}
V(t) \leq\left\|V\left(t_{0}\right)\right\| \exp \left(-g_{1} t\right), \\
V(\sigma T) \leq\left\|V\left(t_{0}\right)\right\| \exp \left(-g_{1} \sigma T\right) .
\end{gathered}
$$

(2) For $\sigma T \leq t<T$,

$$
\begin{aligned}
V(t) & \leq(\|V(\sigma T)\|) \exp \left(g_{2}(t-\sigma T)\right) \\
& \leq\left(\left\|V\left(t_{0}\right)\right\|\right) \exp \left(-g_{1} \sigma T+g_{2}(t-\sigma T)\right), \\
V(T) & \leq\left(\left\|V\left(t_{0}\right)\right\|\right) \exp \left(-g_{1} \sigma T+g_{2}(T-\sigma T)\right) .
\end{aligned}
$$

(3) For $T \leq t<T+\sigma T$,

$$
\begin{aligned}
V(t) \leq & (\|V(T)\|) \exp \left(-g_{1}(t-T)\right) \\
\leq & \left(\left\|V\left(t_{0}\right)\right\|\right) \exp \left(-g_{1} \sigma T+g_{2}(T-\sigma T)-g_{1}(t-T)\right), \\
V(T+\sigma T) & \leq\left(\left\|V\left(t_{0}\right)\right\|\right) \\
& \quad \times \exp \left(-g_{1} \sigma T+g_{2}(T-\sigma T)-g_{1}(T+\sigma T-T)\right) \\
\leq & \left(\left\|V\left(t_{0}\right)\right\|\right) \exp \left(-2 g_{1} \sigma T+g_{2}(T-\sigma T)\right) .
\end{aligned}
$$

(4) For $T+\sigma T \leq t<2 T$,

$$
\begin{gathered}
V(t) \leq\left(\left\|V\left(t_{0}\right)\right\|\right) \\
\quad \times \exp \left(-2 g_{1} \sigma T+g_{2}(T-\sigma T)+g_{2}(t-T-\sigma T)\right), \\
V(2 T) \leq\left(\left\|V\left(t_{0}\right)\right\|\right) \exp \left(-2 g_{1} \sigma T+2 g_{2}(T-\sigma T)\right) .
\end{gathered}
$$

By induction, we have the following.

(5) For $n T \leq t<n T+\sigma T$,

$$
\begin{aligned}
V(t) \leq & (\|V(n T)\|) \exp \left(-g_{1}(t-n T)\right) \\
\leq & \left(\left\|V\left(t_{0}\right)\right\|\right) \exp \left(-g_{1}(t-n T)\right) \\
& \times \exp \left(-n g_{1} \sigma T+n g_{2}(T-\sigma T)\right) \\
\leq & \left(\left\|V\left(t_{0}\right)\right\|\right) \exp \left(-n g_{1} \sigma T+n g_{2}(T-\sigma T)\right) .
\end{aligned}
$$

Note that $(t-\sigma T) / T \leq n<t / T$; in this case, we can obtain

$$
\begin{aligned}
V(t) \leq & \left(\left\|V\left(t_{0}\right)\right\|\right) \\
& \times \exp \left(-\frac{\left(g_{1} \sigma T-g_{2}(T-\sigma T)\right)(t-\sigma T)}{T}\right) \\
\leq & \left(\left\|V\left(t_{0}\right)\right\|\right) \exp \left(-\left(g_{1} \sigma-g_{2}(1-\sigma)\right)(t-\sigma T)\right) .
\end{aligned}
$$

(6) For $n T+\sigma T \leq t<(n+1) T$

$$
\begin{aligned}
V(t) \leq & (\|V(n T+\sigma T)\|) \exp \left(-g_{2}(t-n T-\sigma T)\right) \\
\leq & \left(\left\|V\left(t_{0}\right)\right\|\right) \exp \left(-g_{2}(t-n T-\sigma T)\right) \\
& \times \exp \left(-(n+1) g_{1} \sigma T+g_{2}(n+1)(T-\sigma T)\right) \\
\leq & \left(\left\|V\left(t_{0}\right)\right\|\right) \\
& \times \exp \left(-(n+1) g_{1} \sigma T+g_{2}(n+1)(T-\sigma T)\right) .
\end{aligned}
$$

Note that $t / T \leq n+1<(t+T-\sigma T) / T$; in this case, we can obtain

$$
\begin{aligned}
V(t) \leq & \left(\left\|V\left(t_{0}\right)\right\|\right) \\
& \times \exp \left(-(n+1) g_{1} \sigma T+g_{2}(n+1)(T-\sigma T)\right) \\
\leq & \left(\left\|V\left(t_{0}\right)\right\|\right) \exp \left(-\left(g_{1} \sigma-g_{2}(1-\sigma)\right)(t-\sigma T)\right) .
\end{aligned}
$$

Therefore, for any $t \geq 0$,

$$
\begin{aligned}
\|e(t)\|^{2} & =V(t) \\
& \leq\left(\left\|V\left(t_{0}\right)\right\|\right) \exp \left(-\left(g_{1} \sigma-g_{2}(1-\sigma)\right)(t-\sigma T)\right) .
\end{aligned}
$$

This implies that the projective lag synchronization error system (7) is globally exponentially stable, and the following estimate holds:

$$
\|e(t)\| \leq\left(\sqrt{\left\|V\left(t_{0}\right)\right\|}\right) \exp \left(-\frac{\left(g_{1} \sigma-g_{2}(1-\sigma)\right)(t-\sigma T)}{2}\right) .
$$

This implies that the projective lag synchronization between the master system (2) and slave system (4) is achieved.

Let $g_{1}{ }^{*}=-\lambda_{\max }\left(C+C^{T}\right)+2 k$ and $g_{2}{ }^{*}=\lambda_{\max }\left(C+C^{T}\right)$, where $g_{1}{ }^{*} \geq g_{1}, g_{2}{ }^{*} \leq g_{2}$. If we replace the first condition in Theorem 2 with $g_{1}{ }^{*}, g_{2}{ }^{*}$, then Theorem 2 also can hold. In addition, one can obtain the following corollary from Theorem 2.

Corollary 3. Suppose that there exist positive scalars $k$ and $\sigma$ satisfying $0<\sigma<1$ such that

$$
g_{1}{ }^{*} \sigma-(1-\sigma) g_{2}{ }^{*}>0,
$$

where $g_{1}{ }^{*}=-\lambda_{\max }\left(C+C^{T}\right)+2 k$ and $g_{2}{ }^{*}=\lambda_{\max }\left(C+C^{T}\right)$. Then, the system (7) is exponentially stable, and the projective lag synchronization between the master system (2) and the slave system (4) under intermittent control (5) is achieved.

Remark 4. If $\alpha=1$, it is clear that the lag synchronization between the system (2) and system (4) will occur.

Remark 5. It is clear that when the time delay vanishes, that is, $\theta=0$, we have $e(t)=y(t)-\alpha x(t)$, which implies that the projective synchronization between master system (2) without delay and system (4) without delay will occur. 
Remark 6. From Corollary 3, one observes that the control strength $k$ can be estimated as follows:

$$
k>k^{*}=\frac{\lambda_{\max }\left(C+C^{T}\right)}{2 \sigma}>0 .
$$

Note that $C$ are determined only by the system itself, and $\sigma$ is control parameter. Then, we can estimate the feasible region $D$ of control parameters $(k, \sigma)$ as follows:

$$
D=\left\{(k, \sigma) \mid k>k^{*}=\frac{\lambda_{\max }\left(C+C^{T}\right)}{2 \sigma}>0,0<\sigma<1\right\} .
$$

\section{Numerical Example}

In this section, Lu neural oscillator [17] is presented as an example to verify the effectiveness of Theorem 2. The programs DDE23 in MATLAB are used to solve numerically the delay differential equations.

Example 1. Consider the Lu neural oscillator [17]

$$
\dot{x}(t)=-C x(t)+A f(x(t))+B g(x(t-1)),
$$

where

$$
C=\left(\begin{array}{ll}
1 & 0 \\
0 & 1
\end{array}\right), \quad A=\left(\begin{array}{ll}
3.0 & 5.0 \\
0.1 & 2.0
\end{array}\right), \quad B=\left(\begin{array}{cc}
-2.5 & 0.2 \\
0.1 & -1.5
\end{array}\right),
$$

and $f(x(t))=g(x(t))=\tanh (x(t))$.

This model was investigated by Lu in [17] where it was shown to be chaotic, as shown in Figure 1. The corresponding slave system is given by

$$
\dot{y}(t)=-C y(t)+A f(y(t))+B g(y(t-1))+u(t) .
$$

From Theorem 2, the controller can be obtained as follows:

$$
u(t)=\left\{\begin{array}{c}
-A f(y(t))-B g(y(t-1))+\alpha A f(x(t-\theta)) \\
+\alpha B g(x(t-1-\theta))-k(y(t)-\alpha x(t-\theta)), \\
n T \leq t<n T+\sigma T, \\
-A f(y(t))-B g(y(t-1))+\alpha A f(x(t-\theta)) \\
+\alpha B g(x(t-1-\theta)), \\
n T \leq t<n T+\sigma T .
\end{array}\right.
$$

So, when $n T \leq t<n T+\sigma T$, we have

$$
\begin{aligned}
y(t)= & -C y(t)+A f(y(t))+B g(y(t-1)) \\
& -A f(y(t))-B g(y(t-1)) \\
& +\alpha A f(x(t-\theta))+\alpha B g(x(t-1-\theta))-k e(t) \\
= & -C y(t)+\alpha A f(x(t-\theta)) \\
& +\alpha B g(x(t-1-\theta))-k e(t) .
\end{aligned}
$$

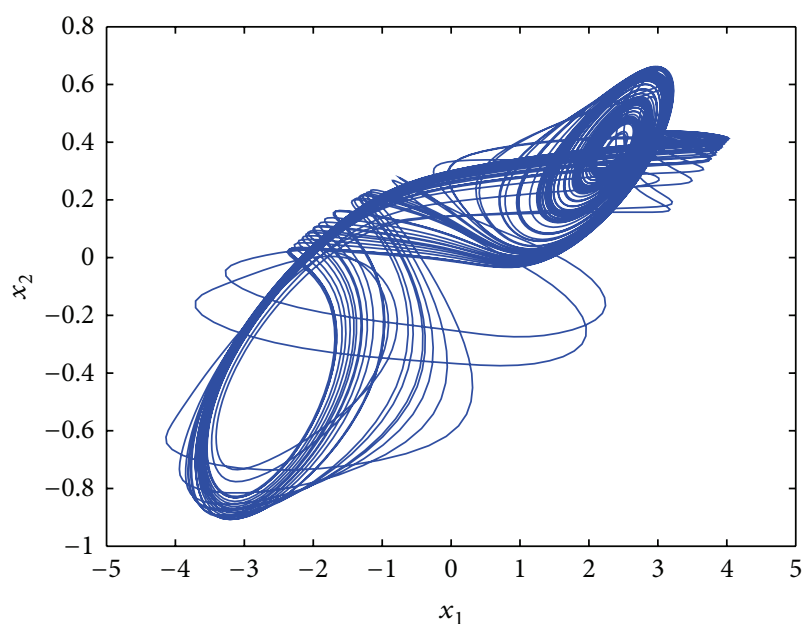

FIgURE 1: The chaotic attractor of the Lu oscillator described by (7) with initial value $x_{1}(\theta)=0.2, x_{2}(\theta)=-0.5$, for $\theta \in[-1,0]$.

When $n T+\sigma T \leq t<(n+1) T$, we have

$$
\begin{aligned}
y(t)= & -C y(t)+A f(y(t))+B g(y(t-1)) \\
& -A f(y(t))-B g(y(t-1)) \\
& +\alpha A f(x(t-\theta))+\alpha B g(x(t-1-\theta)) \\
= & -C y(t)+\alpha A f(x(t-\theta))+\alpha B g(x(t-1-\theta)) .
\end{aligned}
$$

Noting that $\lambda_{\max }\left(C+C^{T}\right)=2$, the feasible region of control parameters $(k, \sigma)$ is $D=\left\{(k, \sigma) \mid k<k^{*}=-1 / \sigma, 0<\right.$ $\sigma<1\}$, as shown in Figure 2. For numerical simulation, we select $\alpha=2, \theta=0.01, \sigma=0.1$, and $k=10$ and plot the norm of projective lag synchronization errors curve, as shown in Figure 3. As the time $t$ goes to infinity, the projective lag synchronization error system is stable. Hence, the projective lag synchronization between system (27) and system (29) is achieved.

\section{Conclusions}

This paper addressed projective lag synchronization of coupled neural networks with time delay. Based on Lyapunov stability theory and adaptive control techniques, several criteria for projective lag synchronization of identical neural networks with time delay have been established. With the proposed method, the simulations of projective lag between coupled Lu systems have showed the effectiveness of theoretical result.

\section{Acknowledgments}

This publication was made possible by NPRP Grant no. NPRP 4-1162-1-181 from the Qatar National Research Fund (a member of Qatar Foundation). The statements made herein are solely the responsibility of the authors. This work was also supported by Natural Science Foundation of China (Grant 


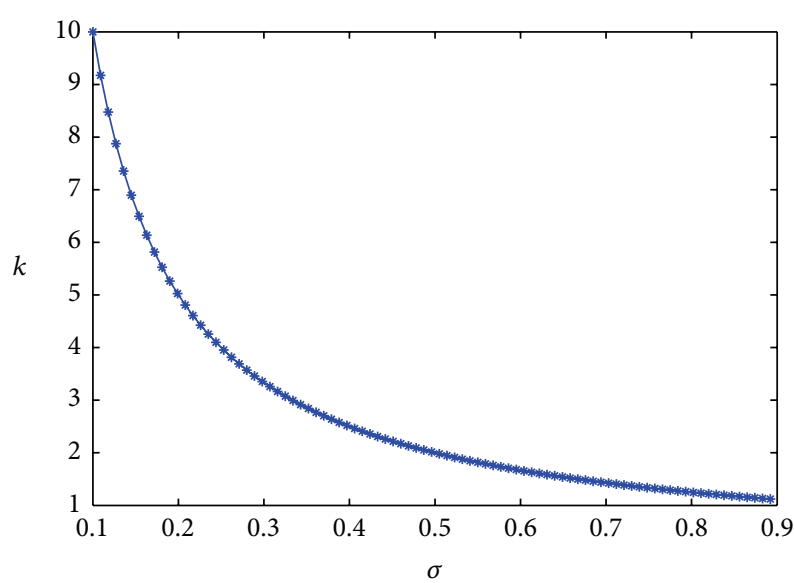

Figure 2: The relationship between $k$ and $\sigma$.

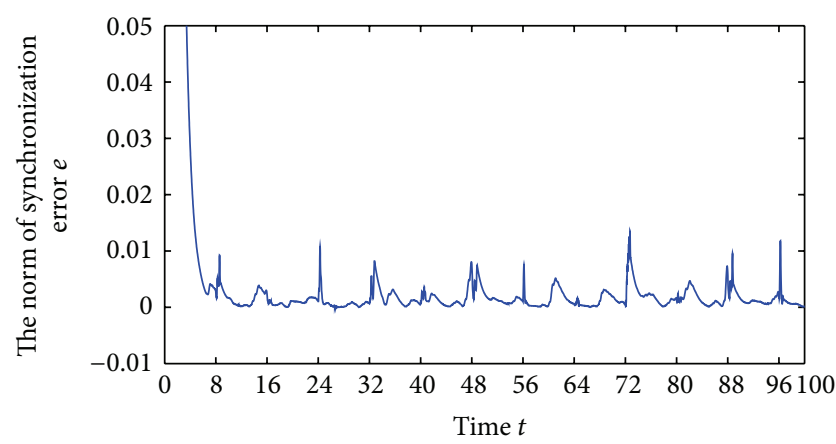

FIGURE 3: The norm of synchronization error curve with $\alpha=2, \theta=$ $0.01, \sigma=0.1$ and $k=10$.

no. 61374078), the Scientific and Technological Research Program of Chongqing Municipal Education Commission (Grant no. KJ121505), the Natural Science Foundation Project of Chongqing CSTC (Grant no. cstc2011jjA40005).

\section{References}

[1] L. M. Pecora and T. L. Carroll, "Synchronization in chaotic systems," Physical Review Letters, vol. 64, no. 8, pp. 821-824, 1990.

[2] L. M. Pecora and T. L. Carroll, "Driving systems with chaotic signals," Physical Review A, vol. 44, no. 4, pp. 2374-2383, 1991.

[3] N. F. Rulkov, M. M. Sushchik, L. S. Tsimring, and H. D. I. Abarbanel, "Generalized synchronization of chaos in directionally coupled chaotic systems," Physical Review E, vol. 51, no. 2, pp. 980-994, 1995.

[4] R. Mainieri and J. Rehacek, "Projective synchronization in three-dimensional chaotic systems," Physical Review Letters, vol. 82, no. 15, pp. 3042-3045, 1999.

[5] M. G. Rosenblum, A. S. Pikovsky, and J. Kurths, "Phase synchronization of chaotic oscillators," Physical Review Letters, vol. 76, no. 11, pp. 1804-1807, 1996.

[6] M. G. Rosenblum, A. S. Pikovsky, and J. Kurths, "From phase to lag synchronization in coupled chaotic oscillators," Physical Review Letters, vol. 78, no. 22, pp. 4193-4196, 1997.
[7] C. Masoller and D. H. Zanette, "Anticipated synchronization in coupled chaotic maps with delays," Physica A, vol. 300, no. 3-4, pp. 359-366, 2001.

[8] X. Li, C. Ding, and Q. Zhu, "Synchronization of stochastic perturbed chaotic neural networks with mixed delays," Journal of the Franklin Institute, vol. 347, no. 7, pp. 1266-1280, 2010.

[9] H. Huang, G. Feng, and J. Cao, "Exponential synchronization of chaotic Lur'e systems with delayed feedback control," Nonlinear Dynamics, vol. 57, no. 3, pp. 441-453, 2009.

[10] D. Zhang and J. Xu, "Projective synchronization of different chaotic time-delayed neural networks based on integral sliding mode controller," Applied Mathematics and Computation, vol. 217, no. 1, pp. 164-174, 2010.

[11] K. S. Sudheer and M. Sabir, "Adaptive function projective synchronization of two-cell Quantum-CNN chaotic oscillators with uncertain parameters," Physics Letters A, vol. 373, no. 21, pp. 1847-1851, 2009.

[12] S. Chen and J. Cao, "Projective synchronization of neural networks with mixed time-varying delays and parameter mismatch," Nonlinear Dynamics, vol. 67, no. 2, pp. 1397-1406, 2012.

[13] L. Chen, Y. Chai, and R. Wu, "Modified function projective synchronization of chaotic neural networks with delays based on observer," International Journal of Modern Physics C, vol. 22, no. 2, pp. 169-180, 2011.

[14] C. Li, X. Liao, and T. Huang, "Exponential stabilization of chaotic systems with delay by periodically intermittent control," Chaos, vol. 17, no. 1, Article ID 013103, 2007.

[15] J. Huang, C. Li, and Q. Han, "Stabilization of delayed chaotic neural networks by periodically intermittent control," Circuits, Systems, and Signal Processing, vol. 28, no. 4, pp. 567-579, 2009.

[16] J. Huang, C. Li, T. Huang, and Q. Han, "Lag quasisynchronization of coupled delayed systems with parameter mismatch by periodically intermittent control," Nonlinear Dynamics, vol. 71, no. 3, pp. 469-478, 2013.

[17] H. Lu, "Chaotic attractors in delayed neural networks," Physics Letters A, vol. 298, no. 2-3, pp. 109-116, 2002. 


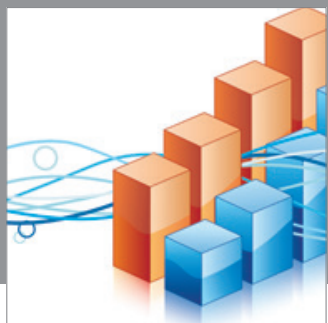

Advances in

Operations Research

mansans

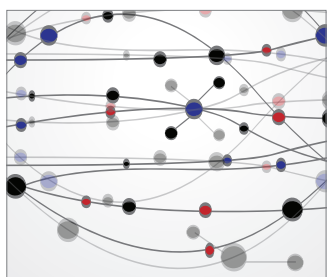

The Scientific World Journal
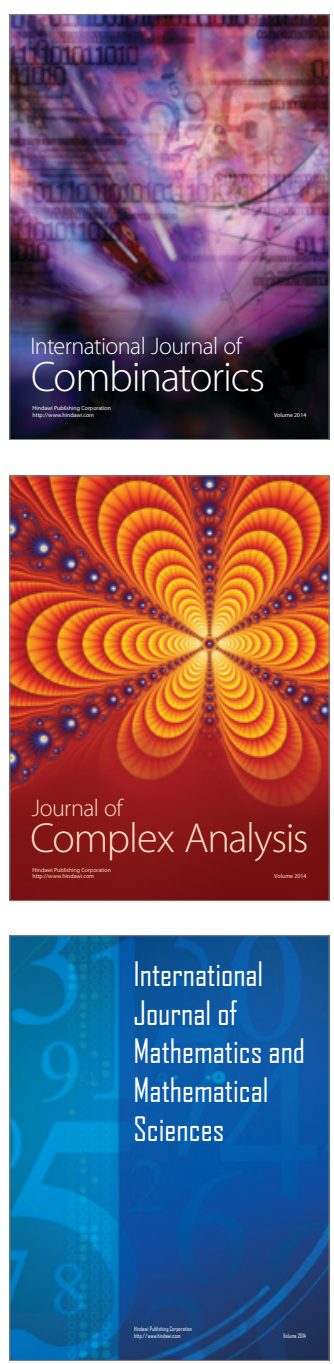
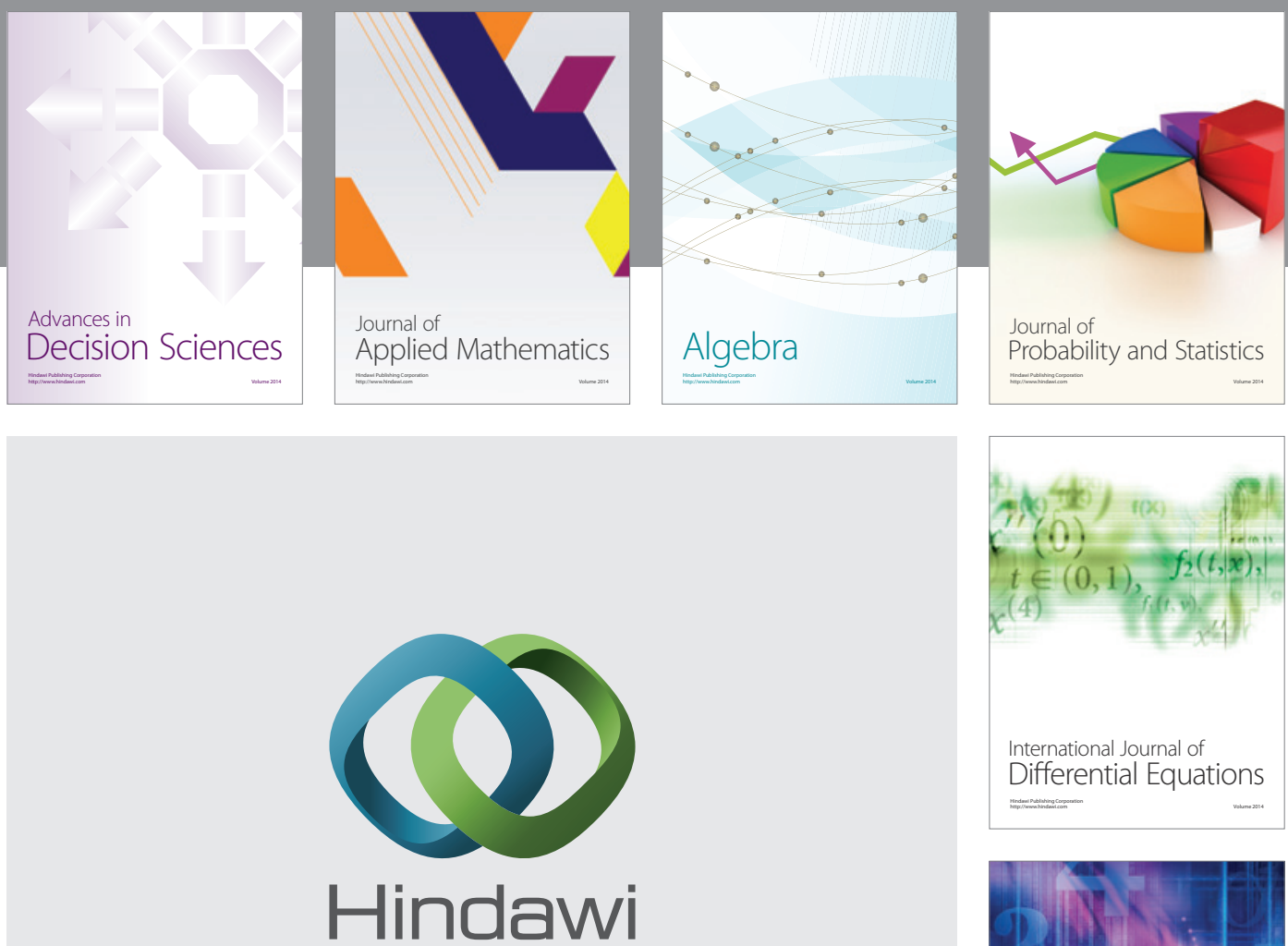

Submit your manuscripts at http://www.hindawi.com
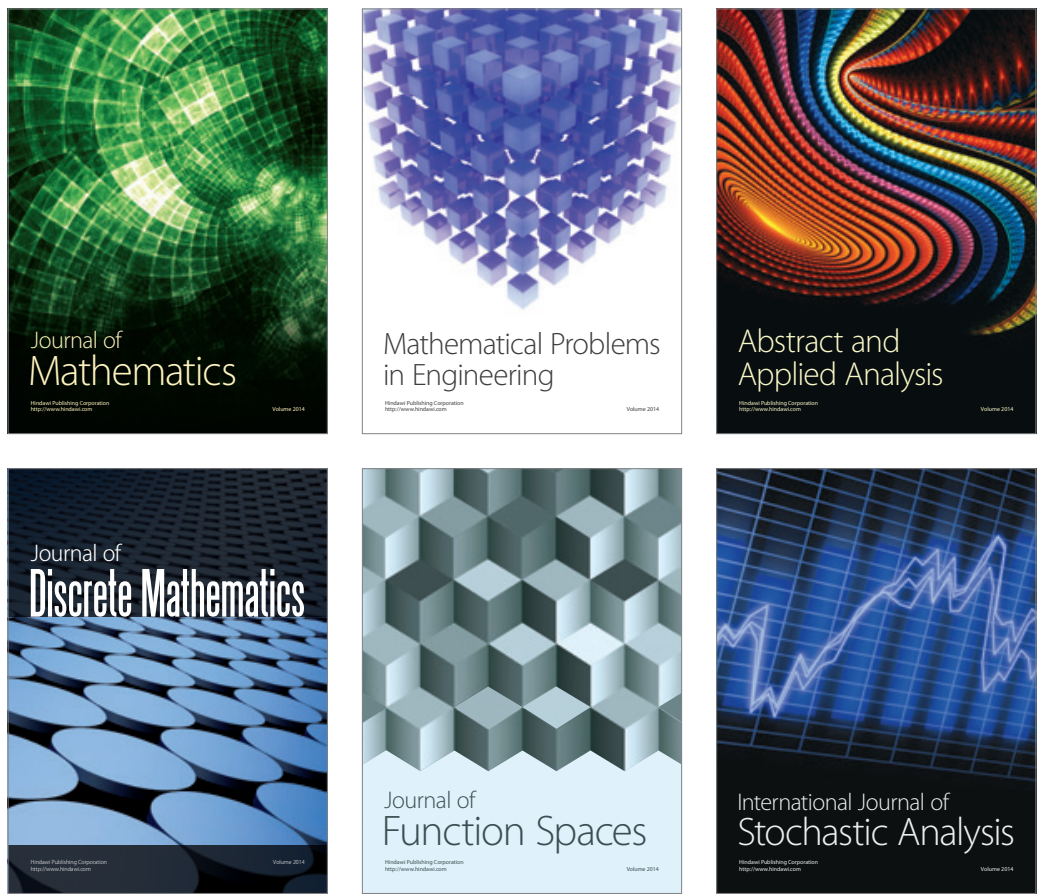

Journal of

Function Spaces

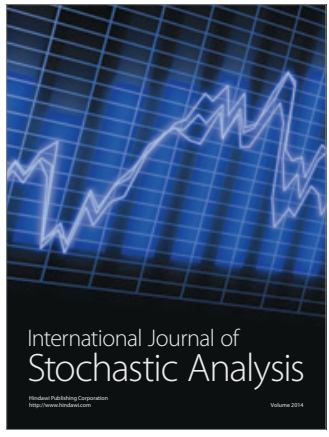

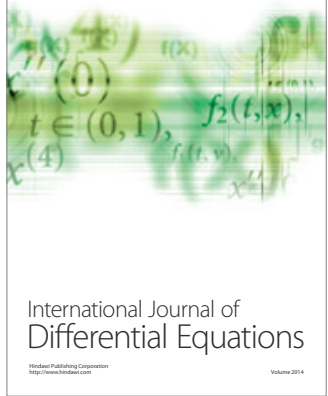
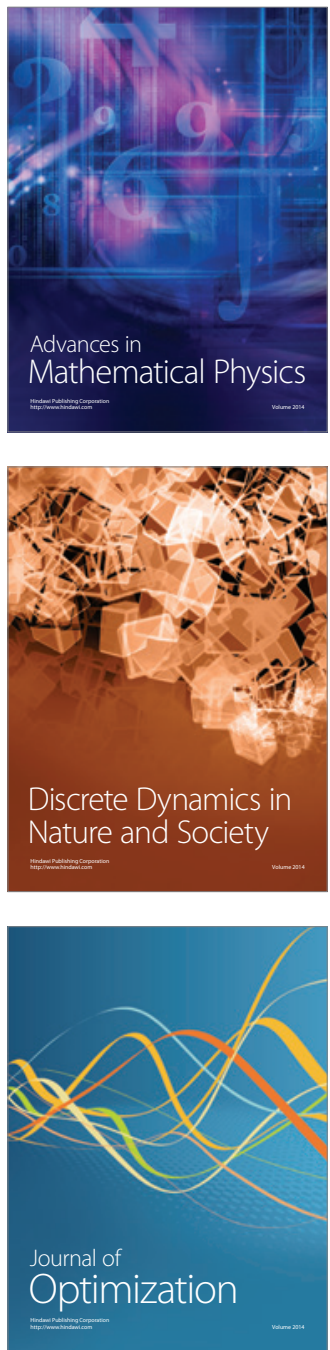